\title{
Antibiotics that target mitochondria effectively eradicate cancer stem cells, across multiple tumor types: Treating cancer like an infectious disease
}

\author{
Rebecca Lamb ${ }^{1,2, *}$, Bela Ozsvari ${ }^{1,2, *}$, Camilla L. Lisanti ${ }^{3, * *}$, Herbert B. Tanowitz ${ }^{4}$, \\ Anthony Howell ${ }^{1,2}$, Ubaldo E. Martinez-Outschoorn ${ }^{5}$, Federica Sotgia ${ }^{1,2}$ and Michael \\ P. Lisanti ${ }^{1,2}$ \\ ${ }^{1}$ The Breakthrough Breast Cancer Research Unit, Institute of Cancer Sciences, University of Manchester, UK \\ ${ }^{2}$ The Manchester Centre for Cellular Metabolism (MCCM), Institute of Cancer Sciences, University of Manchester, UK \\ ${ }^{3}$ The Moor Allerton Preparatory School, Didsbury, Manchester, UK \\ ${ }^{4}$ Departments of Pathology and Medicine, The Albert Einstein College of Medicine, Bronx, NY, USA \\ ${ }^{5}$ The Kimmel Cancer Center, Philadelphia, PA, USA \\ ${ }^{*}$ These authors contributed equally to this study and should be considered as co-first authors \\ ** provided the initial idea for this study \\ Correspondence to: Michael P. Lisanti, email: michaelp.lisanti@gmail.com \\ Federica Sotgia, email: fsotgia@gmail.com \\ Keywords: mitochondria, mitochondrial biogenesis, cancer stem cells, tumor initiating cells, antibiotics \\ Received: December 24, $2014 \quad$ Accepted: January 15, $2015 \quad$ Published: January 22, 2015 \\ This is an open-access article distributed under the terms of the Creative Commons Attribution License, which permits unrestricted use, \\ distribution, and reproduction in any medium, provided the original author and source are credited.
}

\section{ABSTRACT}

Here, we propose a new strategy for the treatment of early cancerous lesions and advanced metastatic disease, via the selective targeting of cancer stem cells (CSCs), a.k.a., tumor-initiating cells (TICs). We searched for a global phenotypic characteristic that was highly conserved among cancer stem cells, across multiple tumor types, to provide a mutation-independent approach to cancer therapy. This would allow us to target cancer stem cells, effectively treating cancer as a single disease of "stemness", independently of the tumor tissue type. Using this approach, we identified a conserved phenotypic weak point - a strict dependence on mitochondrial biogenesis for the clonal expansion and survival of cancer stem cells. Interestingly, several classes of FDA-approved antibiotics inhibit mitochondrial biogenesis as a known "side-effect", which could be harnessed instead as a "therapeutic effect". Based on this analysis, we now show that 4-to-5 different classes of FDA-approved drugs can be used to eradicate cancer stem cells, in 12 different cancer cell lines, across 8 different tumor types (breast, DCIS, ovarian, prostate, lung, pancreatic, melanoma, and glioblastoma (brain)). These five classes of mitochondrially-targeted antibiotics include: the erythromycins, the tetracyclines, the glycylcyclines, an antiparasitic drug, and chloramphenicol. Functional data are presented for one antibiotic in each drug class: azithromycin, doxycycline, tigecycline, pyrvinium pamoate, as well as chloramphenicol, as proof-of-concept. Importantly, many of these drugs are non-toxic for normal cells, likely reducing the side effects of anti-cancer therapy. Thus, we now propose to treat cancer like an infectious disease, by repurposing FDAapproved antibiotics for anti-cancer therapy, across multiple tumor types. These drug classes should also be considered for prevention studies, specifically focused on the prevention of tumor recurrence and distant metastasis. Finally, recent clinical trials with doxycycline and azithromycin (intended to target cancer-associated infections, 


\section{but not cancer cells) have already shown positive therapeutic effects in cancer patients, although their ability to eradicate cancer stem cells was not yet appreciated.}

\section{INTRODUCTION}

Next generation sequencing and many other very sophisticated means of mutational analysis have given us an incredibly detailed view, or "molecular portrait", of the diversity of genetic modifications that occur during the development of human cancers [1-5].

Despite this knowledge of the genomic landscape of cancer, it still remains extremely difficult to identify what are the primary "driver-mutations", in the context of a "sea" of many other genetic changes [1-5]. The emerging picture is that while a few driver-mutations are common to certain specific cancer sub-types, each patient's tumor is fairly unique in its complexity of genetic changes and that several divergent cancer cell clones may also co-exist, within a single tumor [1-5].

This enormous level of detail and genetic complexity makes it extremely difficult to design new diagnostics and targeted-therapeutics, to achieve the goals of personalized medicine. There are numerous examples in clinical practice of how targeting the suspected "driver mutations" has been disappointing. Cancer control is frequently short lived, even when these drugs are proven to be effective. For example, the BRAF inhibitor vemurafenib is approved for the treatment of patients whose melanoma harbors the V600E mutation, which is thought to be a driver mutation $[6,7]$. However, after treatment with vemurafenib, cancer progression occurs within six months in the vast majority of these patients with V600E mutations [6, 7].

Instead, an alternative approach would be to focus on what is common between different tumor types, rather than on what is divergent between different cancers. One common tractable target may be the property of "stemness" in cancer cells.

Recently, Tomasetti and Vogelstein showed that the life-time risk of two-thirds of cancers could simply be accounted for by the number of times that a given tissue's stem cells undergo cell division [8]. This is consistent with the idea that during aging, somatic mutations may accumulate in tissue stem cells, driving the formation of cancer stem cells [8]. They further concluded that these somatic mutations accounted for more cancer cases than either inherited genetic disease or specific environmental risk factors. These observations are also consistent with the idea that cancer is essentially a disease of "stemness" gone awry $[7,8]$.

Based on this rather simple premise, using unbiased quantitative proteomic profiling [9], we have focused on identifying a global phenotypic property of cancer stem cells (CSCs) that could be targeted across multiple tumor types. We have identified this property as a strict dependence on mitochondrial biogenesis, for the anchorage-independent clonal expansion and survival of the CSC population.

Here, we show that 4-to-5 different classes of FDA-approved antibiotics, which inhibit mitochondrial biogenesis as an "off-target" effect, can be used to eradicate cancer stem cells, in 12 different cancer cell lines, across 8 different tumor types (breast, DCIS, ovarian, prostate, lung, pancreatic, melanoma, and glioblastoma (brain)). Thus, future clinical trials for testing the efficacy of these mitochondrially-targeted antibiotics, in multiple cancer types, are now clearly clinically warranted. Overall, the use of generic antibiotics for anti-cancer therapy should significantly reduce the costs of patient care, making treatment more accessible in the developing world.

\section{RESULTS}

\section{Overall approach: Finding an Achilles' heel in cancer stem cells}

Recently, we used an unbiased proteomics approach to identify what makes cancer stem cells relatively unique, as compared with 'bulk' cancer cells [9]. For this purpose, we characterized the proteome of mammo-spheres derived from two different ER(+) breast cancer lines, specifically MCF7 and T47D cells [9]. Interestingly, we observed that MCF7 mammo-spheres show the marked over-expression of $>60$ mitochondrial-related proteins, as compared with monolayers; nine of these mitochondrial proteins were infinitely upregulated in mammo-spheres [9]. These findings are consistent with the idea that cancer stem cells are anabolic and that they may require mitochondrial biogenesis for their survival and proliferative expansion [9].

To test this hypothesis more directly, here we took advantage of the known side effects of specific classes of antibiotics. Because mitochondria evolved from bacteria that were originally engulfed by eukaryotic cells millions of years ago (known as the "endosymbiotic theory of mitochondrial evolution") [10,11], many classes of FDAapproved antibiotics actually target mitochondria, as a mild side-effect, which is well-tolerated in most patients.

More specifically, the erythromycins and chloramphenicol selectively bind to the large subunit of the mitochondrial ribosome and inhibit mitochondrial biogenesis, by preventing the translation of mitochondrial proteins, mainly related to the mitochondrial OXPHOS complexes (Figure 1A). Similarly, the tetracyclines and glycylcyclines both bind with high affinity to the small subunit of the mitochondrial ribosome and inhibit mitochondrial biogenesis as well (Figure 1A). 
Thus, these four large classes of antibiotics all function as known inhibitors of mitochondrial biogenesis in mammalian cells. This would allow us to use these antibiotics as "investigational tools" to assess if mitochondrial biogenesis is absolutely required for the survival and propagation of cancer stem cells. If successful, these FDA-approved antibiotics could then be re-purposed for the treatment of cancer, to achieve the eradication or more effective elimination of cancer stem cells (Figure 1B).

If mitochondrial biogenesis is indeed required for the propagation of all cancer stem cells, then this new therapeutic approach could be applied across multiple cancer types, perhaps in a mutation independent fashion. In essence, we would be treating cancer based instead on a common global phenotypic property that is characteristic

A

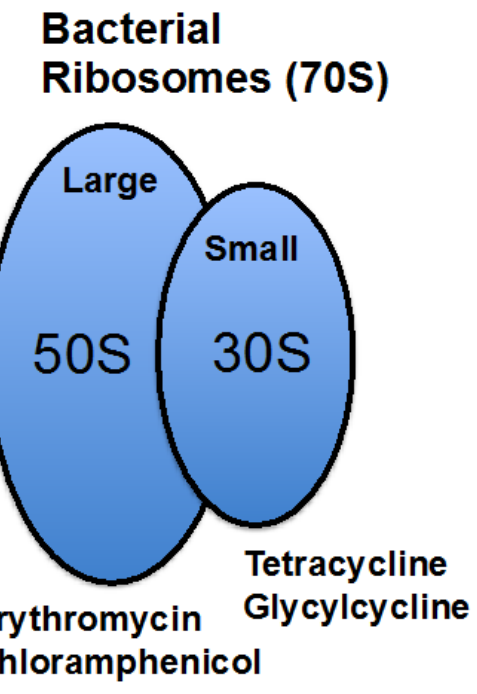

Primary Target

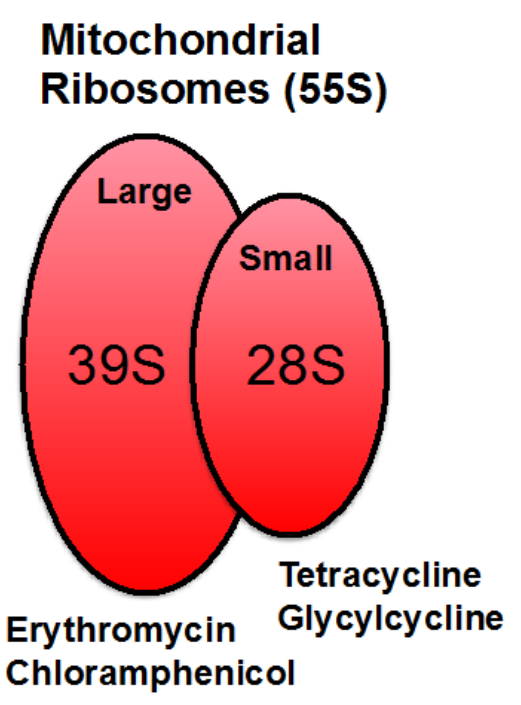

Manageable Side-Effects

B

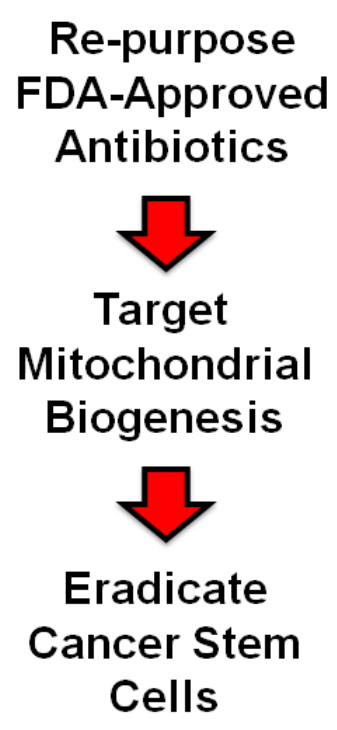

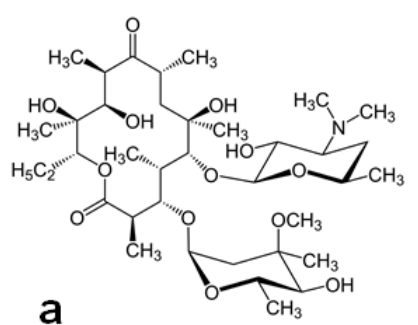

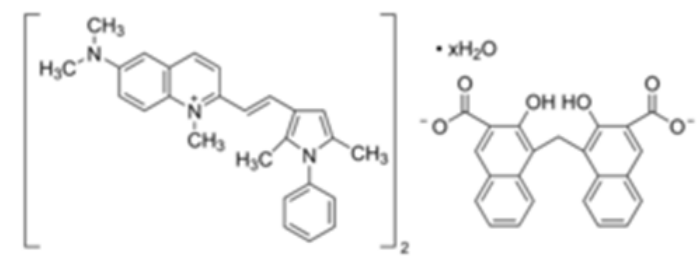

C b<smiles>CN(C)C1C(O)=C(C(N)=O)C(=O)[C@]2(O)C(O)=C3C(=O)c4c(O)cccc4[C@@](C)(O)[C@H]3C[C@H]12</smiles>

Figure 1: Treating cancer like an infectious disease, with antibiotics. Recently, using unbiased proteomics analysis, we showed that mitochondrial proteins were highly upregulated in MCF7 and T47D tumor-spheres, as directly compared with monolayer cells. Thus, we set out to test the hypothesis that tumor-sphere formation was strictly dependent on mitochondrial biogenesis. Interestingly, several known classes of FDA-approved antibiotics function as inhibitors of mitochondrial biogenesis, which results in manageable sideeffects. (A) Bacterial and mitochondrial ribosomes are closely related. Erythromycins and chloramphenicol target the large mitochondrial ribosome, while tetracyclines and glycylcyclines target the small mitochondrial ribosomes, because of conserved similarities with bacterial ribosomes. (B) Examples of FDA-approved antibiotics are shown. The structures of erythromycin (a) and tetracycline (b) are shown, along with pyrvinium pamoate (c). Here, we tested the hypothesis that these different classes of FDA-approved antibiotics could be re-purposed for the targeting of mitochondrial biogenesis and the eradication of cancer stem cells. 
Table 1: Twelve Cancer Cell Models with Broad Applicability

\begin{tabular}{|l|l|}
\hline Cancer Types & Cell Lines \\
\hline Breast (ER+) & $\begin{array}{l}\text { MCF7 } \\
\text { T47D }\end{array}$ \\
\hline Breast (ER-) & MDA-MB-231 \\
\hline DCIS & MCF10.DCIS.com ("pre-malignant") \\
\hline Ovarian & SKOV3 \\
& Tov21G \\
\hline Prostate & PC3 \\
\hline Pancreatic & MIA PaCa2 \\
\hline Lung & A549 \\
\hline Melanoma & A375 \\
\hline Glioblastoma & U-87 MG \\
\hline
\end{tabular}

12 Cell Lines Representing 8 Different Types of Cancer.

of cancer stem cells, allowing this approach to be more broadly applied perhaps to any cancer type (Figure 2).

\section{The Erythromycins: Azithromycin as an example}

Azithromycin is a macrolide antibiotic that is currently used for the treatment of many types of bacterial infections. Azithromycin is a derivative of erythromycin, and is generally more potent and is more slowly eliminated than erythromycin, allowing infections to be treated relatively quickly, over 3-to-5 days. Overall, it shows broad-spectrum anti-bacterial activity. Mechanistically,

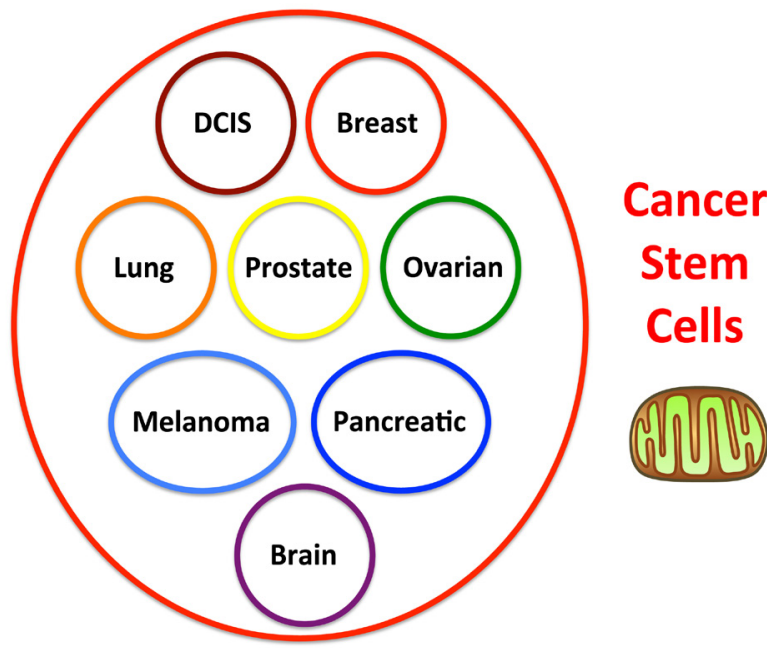

Figure 2: Treating cancer phenotypically as a single disease of increased "stemness": Developing a mutation-independent approach to cancer therapy. One idea is that we could potentially treat cancer as a single disease, if we could identify a global phenotypic characteristic that is conserved across multiple cancer types. For this purpose, we focused on tumor-initiating cells (TICs), a.k.a., cancer stem cells (CSCs), as they share many common properties, such as anchorage-independent growth, i.e., tumor-sphere formation under low-attachment conditions. This global phenotypic property appears to be functionally dependent on increased mitochondrial biogenesis. azithromycin inhibits bacterial growth by preventing protein synthesis. Azithromycin directly binds to the $50 \mathrm{~S}$ subunit of the bacterial ribosome, and specifically inhibits the translation of mRNA species into protein. Importantly, the 50S bacterial ribosome is homologous the 39S mitochondrial ribosome. In fact, many of the bacterial ribosomal subunits have directly-related mitochondrial homologues in eukaryotic cells. As such, erythromycinrelated antibiotics, e.g., azithromycin, behave as inhibitors of mitochondrial biogenesis.

Thus, as a first step, we chose to evaluate the efficacy of azithromycin. For this purpose, azithromycin was tested for its ability to inhibit mammo-sphere formation using MCF7 and T47D cells, over a range of concentrations. Interestingly, in MCF7 cells, azithromycin inhibited mammo-sphere formation with an IC-50 of 50 $\mu \mathrm{M}$; similar results were also obtained in T47D cells (Figure 3).

As a consequence of these positive findings, we next tested the ability of azithromycin to inhibit tumor-sphere formation in a wide-variety of cell lines derived from many different tumor types, including ER(-) breast cancer, ovarian, lung, pancreatic, and prostate cancer, as well as melanoma (summarized in Table 1). For simplicity, we used a single concentration of $250 \mu \mathrm{M}$. Figure 4 (panels A and B) directly shows that azithromycin inhibited tumorsphere formation in these 8 additional well-established cell lines, representing 6 different cancer types. Thus,

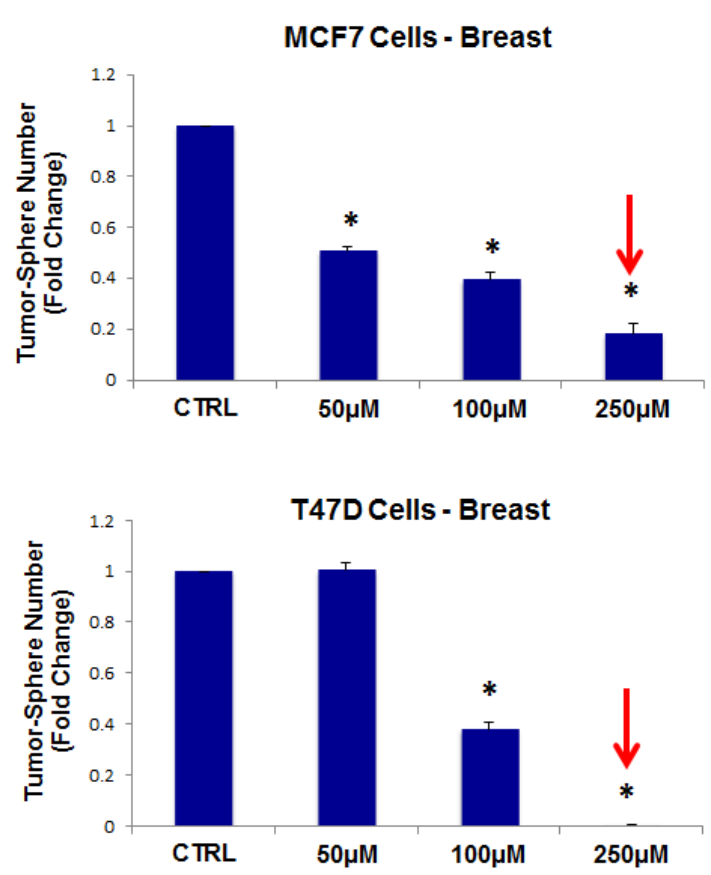

Figure 3: Azithromycin dose-dependently inhibits tumor-sphere formation in MCF7 and T47D cells, two commonly used $\mathbf{E R}(+)$ breast cancer cell lines. Azithromycin was initially tested over the range of $50 \mu \mathrm{M}$ to $250 \mu \mathrm{M}$. Note that $250 \mu \mathrm{M}$ was the most effective. $(*) \mathrm{p}<0.001$. 
azithromycin was effective against tumor-sphere formation in all 10 cell lines tested.

\section{The Tetracyclines: Doxycycline provides proof-of- concept}

Tetracycline is a broad-spectrum antibiotic that is commonly used for the treatment many bacterial infections, and functions as an inhibitor of protein synthesis in bacteria. Today, it is mainly used for the treatment of acne. However, the term "tetracyclines" is also used to describe a class of related semi-synthetic derivatives, that all contain the same chemically-conserved four-member ring structure, that is characteristic of tetracycline. Tetracyclines show bacterio-static activity against nearly all aerobic and anaerobic bacteria, including both Grampositive and Gram-negative types. Tetracyclines inhibit protein synthesis by preventing the binding of activated aminoacyl-tRNAs to the A-site on the 30S subunit of bacterial ribosomes. As such, they reversibly inhibit the addition of new amino acids to the growing polypeptide chain, during protein synthesis. Importantly, the $30 \mathrm{~S}$ bacterial ribosome is homologous the $28 \mathrm{~S}$ mitochondrial

\section{A}
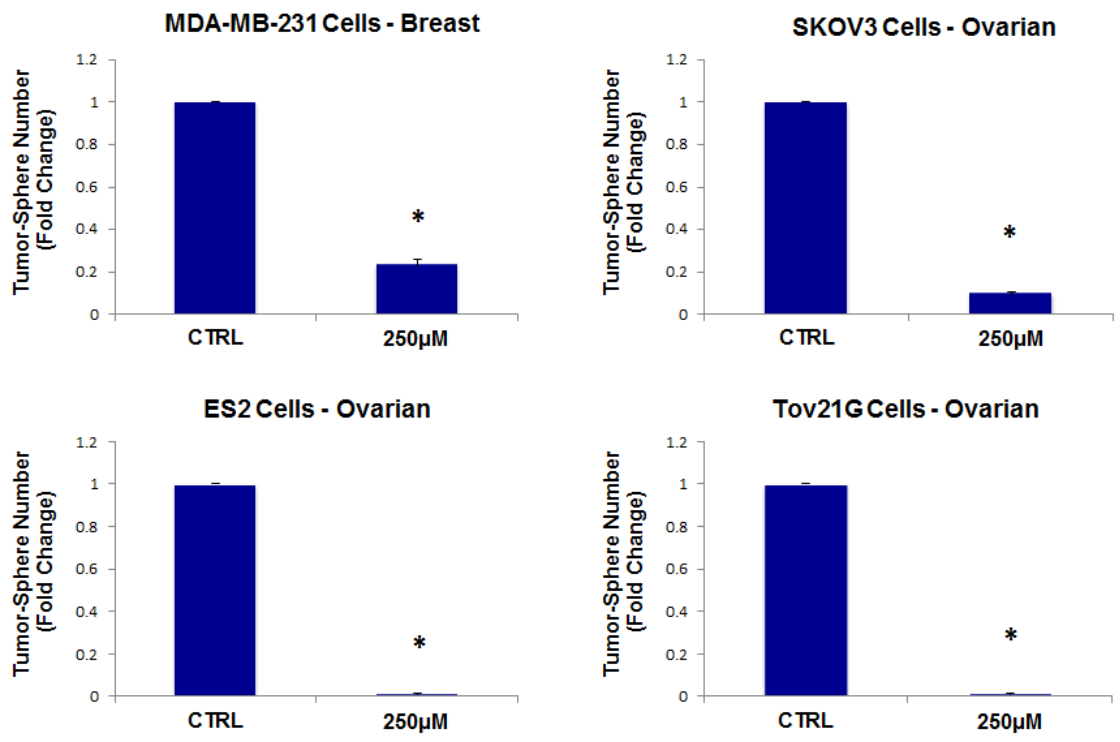

B
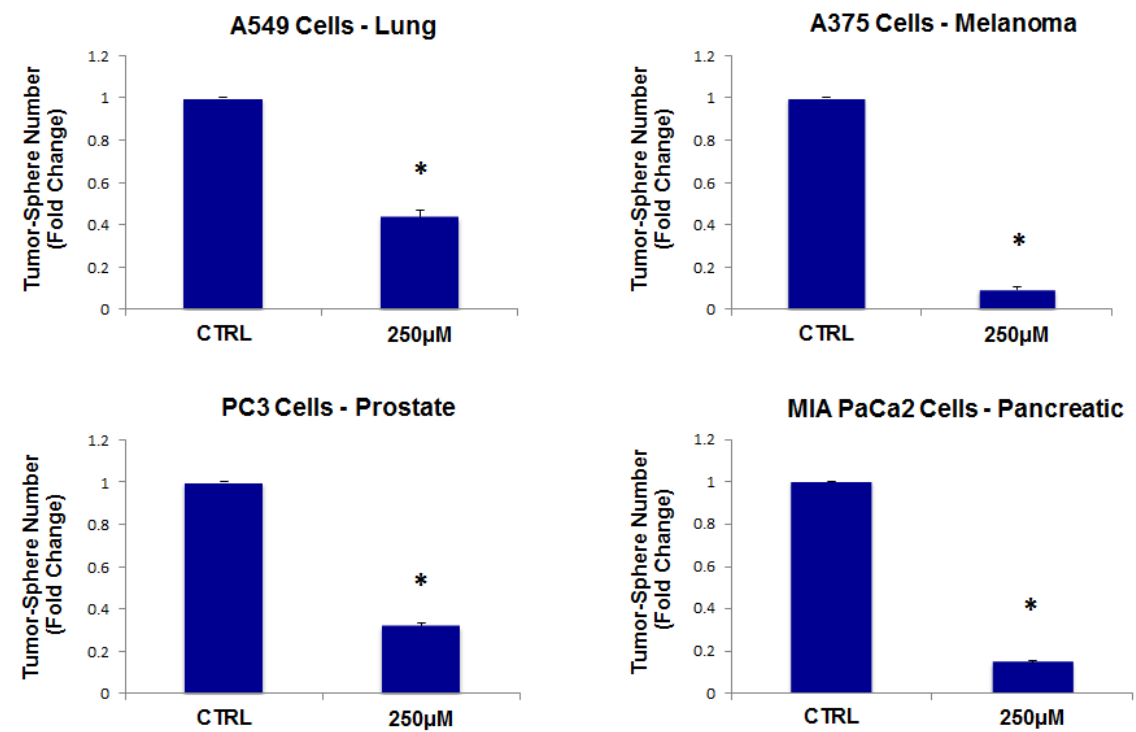

Figure 4: Azithromycin inhibits tumor-sphere formation in eight other cell lines, derived from diverse cancer types. For simplicity, the efficacy of azithromycin was tested at a concentration of $250 \mu \mathrm{M}$. (*)p $<0.001$. (A) ER(-) breast [MDA-MB-231] and ovarian cancer cell lines [SKOV3, ES2, Tov21G] (B) Lung [A549], prostate [PC3], melanoma [A375], and pancreatic [MIA PaCa2]. 
ribosome, accounting for the manageable side-effects of the tetracyclines. As a consequence, tetracycline-based antibiotics are inhibitors of mitochondrial biogenesis. Doxycycline is a tetracycline-derivative with markedly improved efficacy and stability, which was first FDAapproved in the late 1960 s, nearly 50 years ago now.

Thus, we next evaluated the efficacy of the tetracycline-based antibiotic, doxycycline. More specifically, doxycycline was tested for its ability to inhibit mammo-sphere formation using MCF7 and T47D cells, over a large range of concentrations, from $1 \mu \mathrm{M}$ to 250 $\mu \mathrm{M}$. Interestingly, in MCF7 cells, doxycycline inhibited mammo-sphere formation with an IC-50 between 2 and 10 $\mu \mathrm{M}$; virtually identical results were also obtained in T47D cells (Figure 5).

We then tested the ability of doxycycline to inhibit tumor-sphere formation in a broad panel of cancer cell lines derived from many different tumor types (Table 1). For simplicity, we used a single concentration of $50 \mu \mathrm{M}$. Figure 6 (panels A and B) directly shows that doxycycline inhibited tumor-sphere formation in all of these wellestablished cell lines. Thus, doxycycline was effective against tumor-sphere formation in all 10 cell lines tested, across 6 different cancer types.
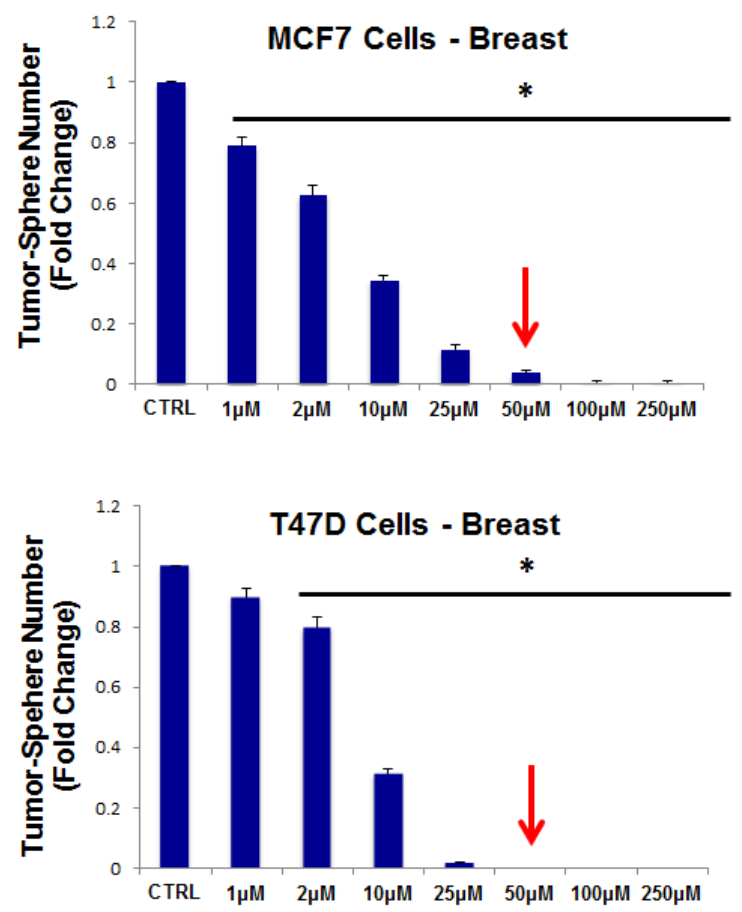

Figure 5: Doxycycline dose-dependently inhibits tumor-sphere formation in MCF7 and T47D cells, two commonly used $\mathbf{E R}(+)$ breast cancer cell lines. Doxcycline was initially tested over the range of $1 \mu \mathrm{M}$ to 250 $\mu \mathrm{M}$. Note that 50 -to- $100 \mu \mathrm{M}$ was the most effective. $(*) \mathrm{p}<0.001$.

\section{The Glycylcyclines: Tigecycline shows efficacy}

The glycylcyclines are a relatively new antibiotic class that are highly-related to the tetracyclines. The glycylcyclines were designed to specifically overcome tetracycline resistance. They have essentially the same mechanism of action as the tetracyclines, as they prevent bacterial protein synthesis. Both tetracyclines and glycylcyclines bind to the $30 \mathrm{~S}$ bacterial ribosomal subunit, thereby inhibiting the binding of a given aminoacyl-tRNA to the A-site of the ribosome. Importantly, it appears that glycylcyclines bind more tightly to the ribosome, than the tetracyclines. Currently, tigecycline is the only FDAapproved glycylcycline. Similarly, the glycylcyclines are inhibitors of mitochondrial biogenesis.

Interestingly, quantitatively similar results were obtained with tigecycline, as compared with doxycycline, showing that it also has the capacity to inhibit tumor-sphere formation, across all 10 cell lines tested. Tigecycline was first tested for its ability to inhibit mammo-sphere formation using MCF7 and T47D cells, over a concentration range, from $10 \mu \mathrm{M}$ to $50 \mu \mathrm{M}$. Interestingly, in both MCF7 and T47D cells, tigecycline inhibited mammo-sphere formation with an IC-50 between 10 and $25 \mu \mathrm{M}$ (Figure 7). For simplicity, we used a single concentration of $50 \mu \mathrm{M}$, in all eight other cell lines, which significantly inhibited tumor-sphere formation (Figures 8).

\section{Anti-parasitc drugs: Pyrvinium pamoate}

Pyrvinium is a cyanine dye, which is an FDAapproved anti-helmintic drug, that has been used to treat pinworms, as well as strongyloidiasis in humans. It was first approved by the FDA in 1955 for the treatment of enterobiasis, and is known to act as an inhibitor of mitochondrial oxidative phosphorylation (OXPHOS), under both normoxia and hypoxic conditions. Several forms of pyrvinium have been prepared, with different anions. Here, we tested the efficacy of pyrvinium pamoate. We used this approach to further validate that mitochondrial function was indeed critical for the survival and propagation of cancer stem cells.

For this purpose, pyrvinium pamoate was tested for its ability to inhibit mammo-sphere formation using MCF7 and T47D cells, over a range of concentrations, from $1 \mathrm{nM}$ to $500 \mathrm{nM}$. Interestingly, in both MCF7 and T47D cells, pyrvinium pamoate inhibited mammo-sphere formation with an IC-50 between $\sim 10$-to-50 $\mathrm{nM}$ (Figure 9).

As such, we next tested the ability of pyrvinium pamoate to inhibit tumor-sphere formation in a widevariety of cell lines derived from many different tumor types (listed in Table 1); we used two concentrations of $250 \mathrm{nM}$ and $500 \mathrm{nM}$. Figure 10 (panels A and B) illustrates that pyrvinium pamoate inhibited tumor-sphere formation across the entire cell line panel; pyrvinium pamoate was 
A

MDA-MB-231 Cells - Breast

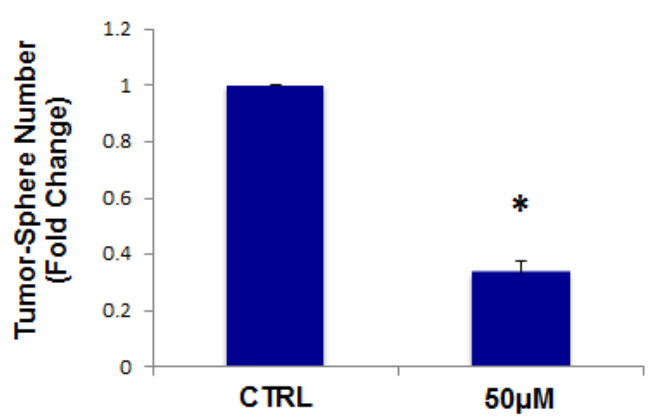

SKoV3 Cells - Ovarian

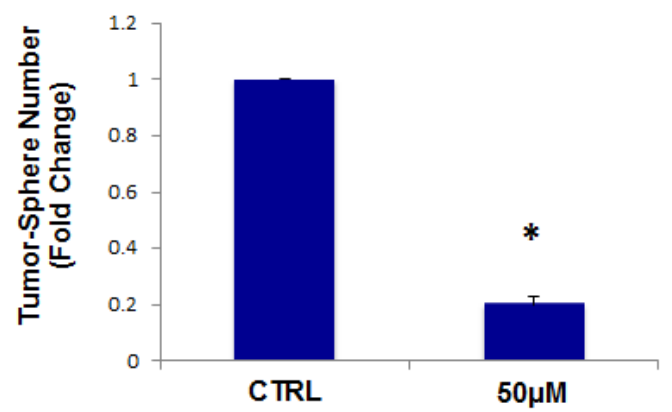

ES2 Cells - Ovarian

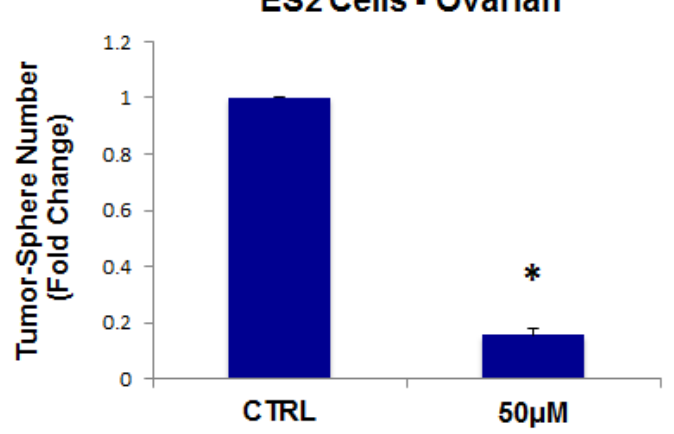

B

A549 Cells - Lung
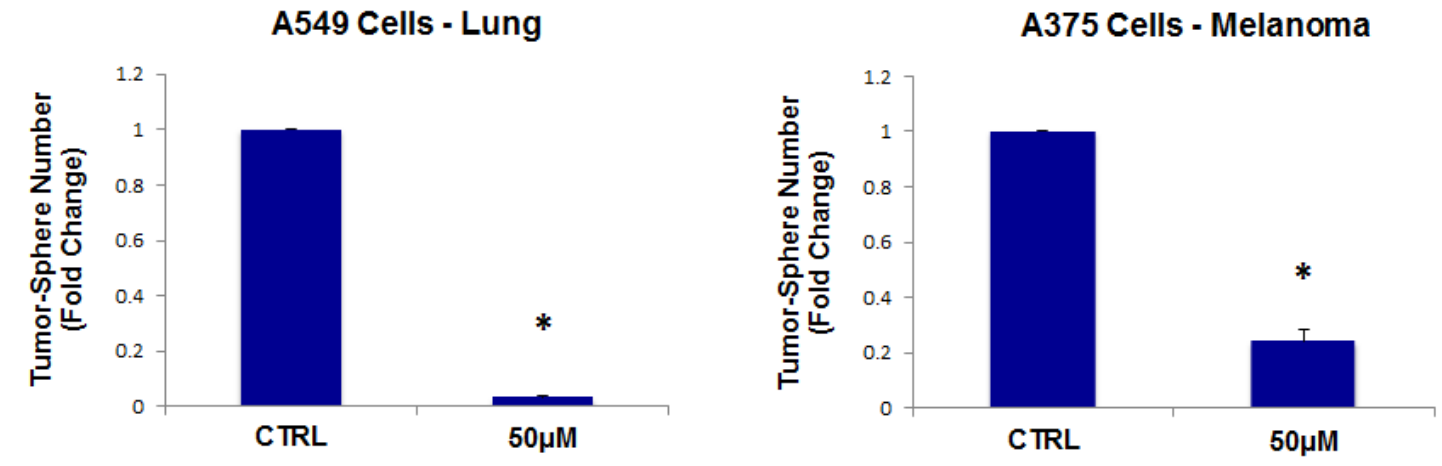

PC3 Cells - Prostate

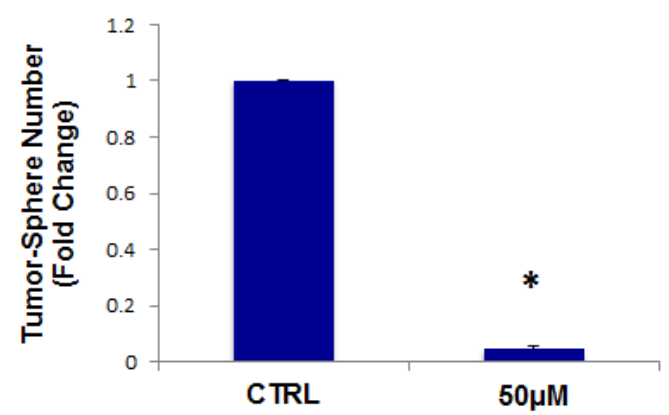

Tov21G Cells - Ovarian
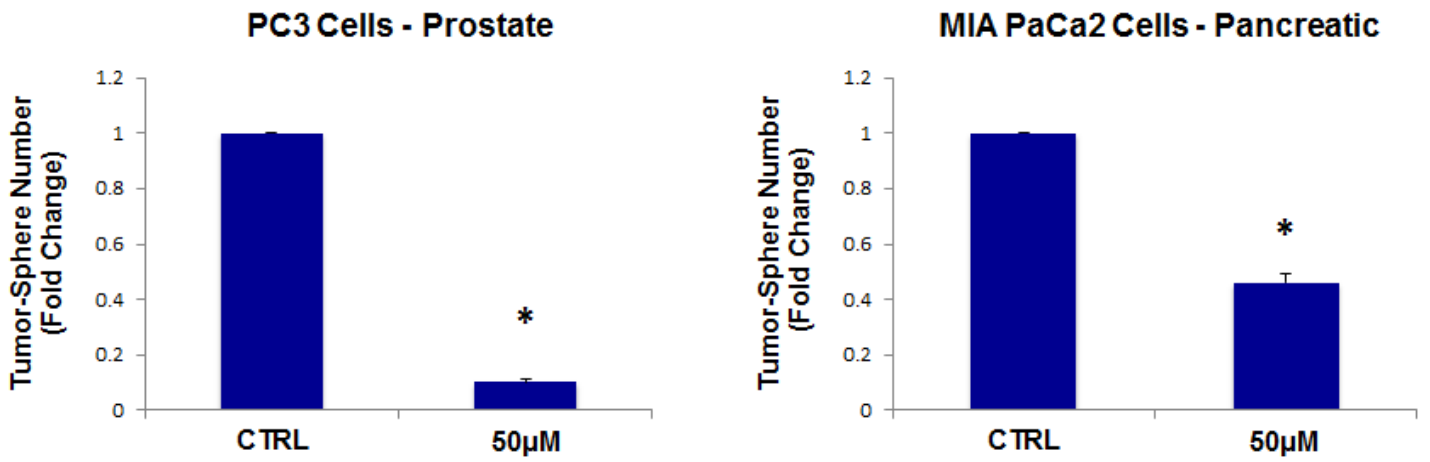

Figure 6: Doxycycline inhibits tumor-sphere formation in eight other cell lines, derived from diverse cancer types. For simplicity, the efficacy of doxycycline was tested at a concentration of $\left.50 \mu \mathrm{M} .{ }^{*}\right) \mathrm{p}<0.001$. (A) ER(-) breast [MDA-MB-231] and ovarian cancer cell lines [SKOV3, ES2, Tov21G] (B) Lung [A549], prostate [PC3], melanoma [A375], and pancreatic [MIA PaCa2]. 
effective against tumor-sphere formation in all 10 cell lines tested, in the nano-molar range.

\section{Efficacy of four classes of antibiotics against DCIS and Glioblastoma CSCs}

To further assess the efficacy of these four classes of antibiotics, we also determined their effectiveness in inhibiting tumor-sphere formation using two other wellestablished cell lines. One was a DCIS-based human cell line, namely MCF10.DCIS.com, which is often used to model pre-malignant mammary lesions in breast cancer patients. The other was derived from one of the most malignant and aggressive tumor types, glioblastoma (brain cancer; U-87 MG).

Figure 11 (panels A and B) shows that all four classes of antibiotics significantly inhibited tumor-sphere formation in both DCIS.com and U-87 MG cells. Thus, our approach can also be used to target both pre-malignant lesions, such as DCIS, or even the most invasive and aggressive cancer types, such as glioblastoma.

Importantly, azithromycin, doxycycline, and tigecycline are all known to cross the blood-brain barrier, making the treatment of brain cancer with these antibiotics feasible.
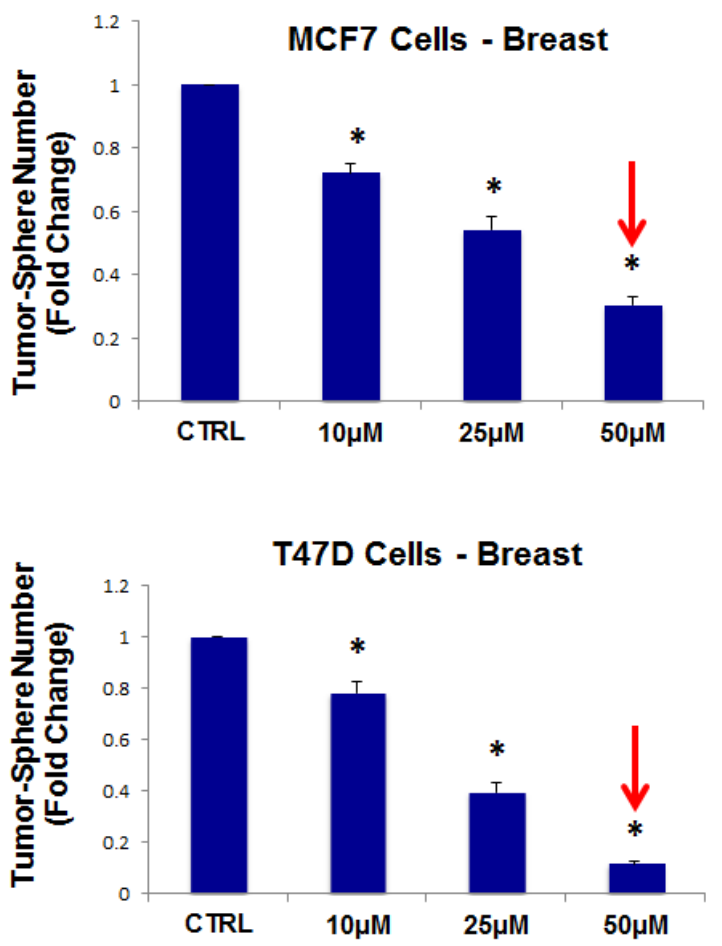

Figure 7: Tigecycline dose-dependently inhibits tumor-sphere formation in MCF7 and T47D cells, two commonly used ER(+) breast cancer cell lines. Tigecycline was initially tested over the range of $10 \mu \mathrm{M}$ to 50 $\mu \mathrm{M}$. Note that $50 \mu \mathrm{M}$ was the most effective. $\left({ }^{*}\right) \mathrm{p}<0.001$.

\section{Evaluating possible toxicity in "bulk" cancer cells and normal fibroblasts}

To evaluate the possible toxicity of our approach on "bulk" cancer cells and normal fibroblasts, we next used i) MCF7 cell monolayers and ii) hTERT-immortalized skin fibroblasts, and assessed their viability using the MTS assay. For these experiments, we focused on two drugs, namely doxycycline and pyrvinium pamoate.

For doxycycline, we used a concentration range from $50 \mu \mathrm{M}$ to $500 \mu \mathrm{M}$. Note that there was little or no toxicity observed in MCF7 cell monolayers or hTERTBJ1 fibroblasts, over this entire range (Figure 12, panels A and B). Importantly, $50 \mu \mathrm{M}$ doxycycline reduced MCF7 mammosphere formation by $>95 \%$ (Figure 5 ). Thus, there is no toxicity for MCF7 cell monolayers or normal fibroblasts at a concentration that nearly completely eliminates CSC expansion (and even at 10X times higher).

For pyrvinium pamoate, we used a concentration range from $500 \mathrm{nM}$ to $5 \mu \mathrm{M}$. Importantly, there was little or no toxicity observed in MCF7 cell monolayers or hTERT-BJ1 fibroblasts (Figure 13, panels A and B). Interestingly, $500 \mathrm{nM}$ pyrvinium pamoate reduced MCF7 mammosphere formation by $>99 \%$ (Figure 9). As such, there is little or no toxicity for MCF7 cell monolayers or normal fibroblasts at a concentration that eliminates CSC expansion (and even at 10X times higher).

Importantly, we observed very limited toxicity, which is consistent with fact that these are well-tolerated antibiotics that are already FDA-approved for patient therapy, but are normally used in the context of infectious disease.

\section{Chloramphenicol}

Chloramphenicol is a bacterio-static antibiotic used for the treatment of a number of bacterial infections, which first became available in the late 1940s. It is a typical broad-spectrum antibiotic. Like the eyrthromycins and the tetracyclines, it functions as an inhibitor of protein synthesis, by binding to the $50 \mathrm{~S}$ bacterial ribosomal subunit, thereby inhibiting peptide bond formation. More specifically, it inhibits peptidyl-transferase activity, preventing protein chain elongation. Similarly, in mammalian cells, chloramphenicol behaves an inhibitor of mitochondrial biogenesis.

Thus, we chose to evaluate the efficacy of chloramphenicol and determined its ability to inhibit mammo-sphere formation using MCF7 cells, over a range of concentrations from $10 \mu \mathrm{M}$ to $1 \mathrm{mM}$. Interestingly, in MCF7 cells, chloramphenicol inhibited mammo-sphere formation with an IC-50 of $\sim 200 \mu \mathrm{M}$ (Figure 14). As such, we have now shown that four independent antibiotic inhibitors of mitochondrial biogenesis (azithromycin, doxycycline, tigecycline, and chloramphenicol) all 
A
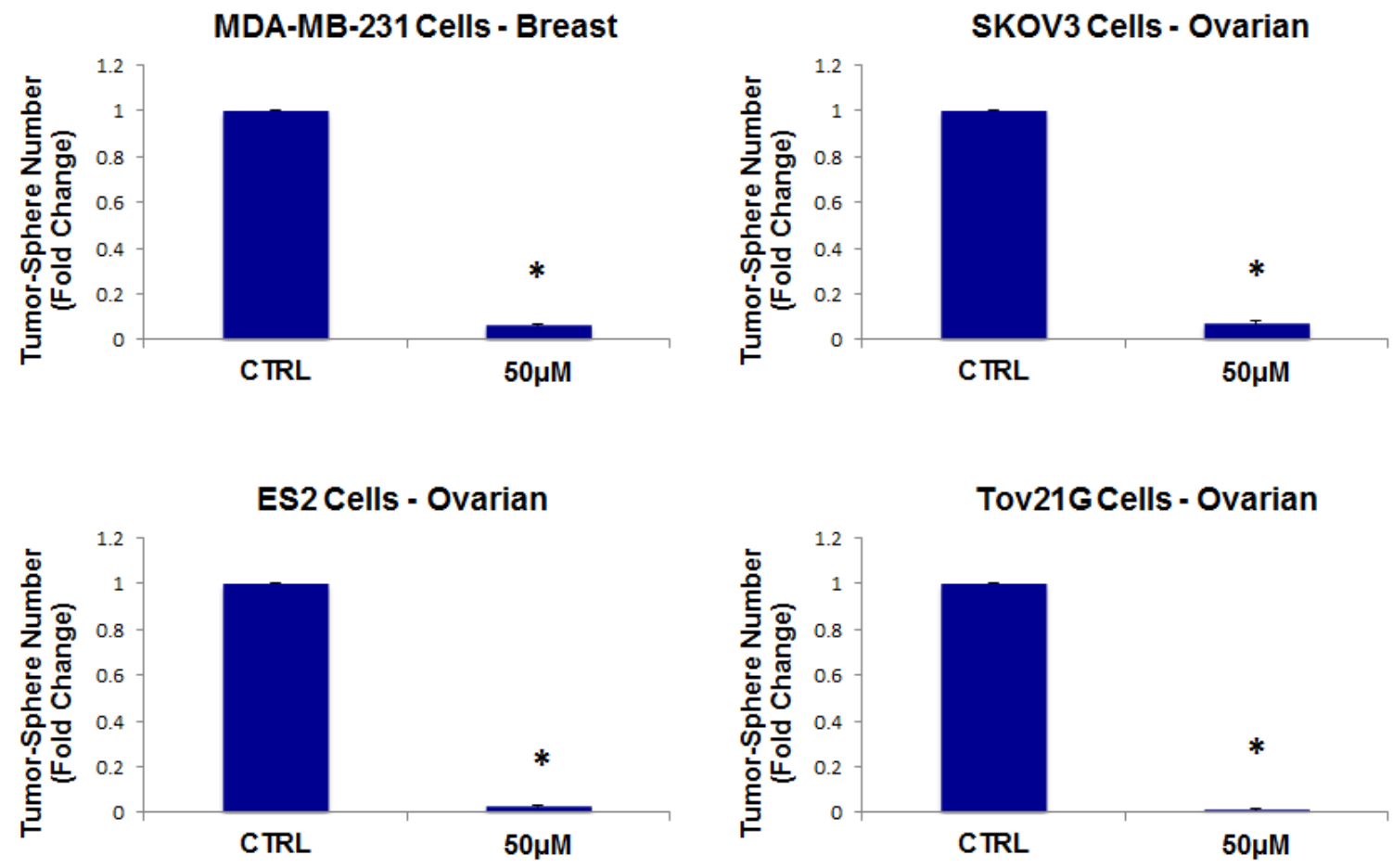

B
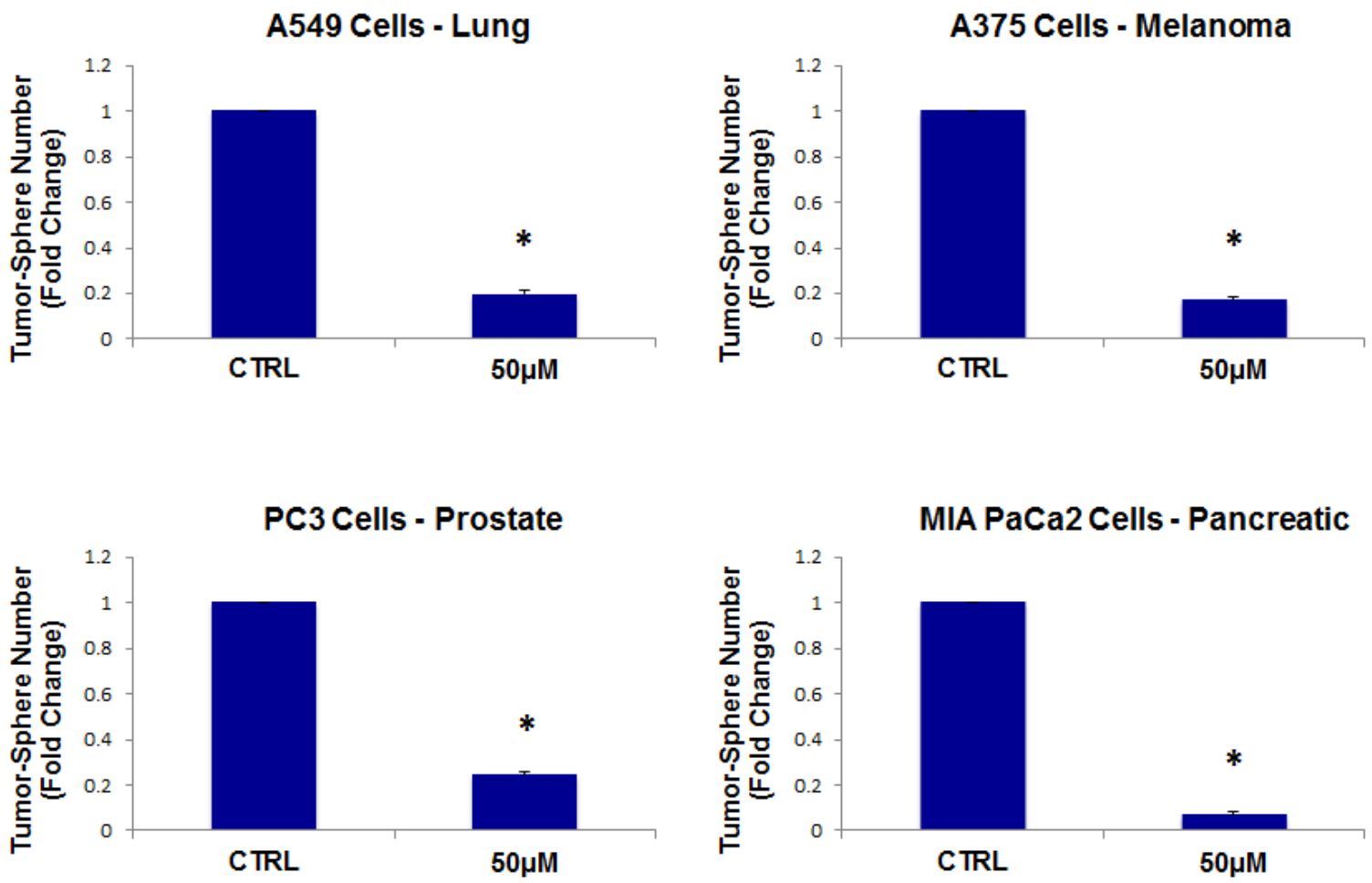

Figure 8: Tigecycline inhibits tumor-sphere formation in eight other cell lines, derived from diverse cancer types. For simplicity, the efficacy of tigecycline was tested at a concentration of $50 \mu \mathrm{M} .\left(^{*}\right) \mathrm{p}<0.001$. (A) ER(-) breast [MDA-MB-231] and ovarian cancer cell lines [SKOV3, ES2, Tov21G] (B) Lung [A549], prostate [PC3], melanoma [A375], and pancreatic [MIA PaCa2]. 
effectively inhibit mammo-sphere formation. However, chloramphenicol was the least potent of the mitochondrial inhibitors that we tested.

\section{DISCUSSION}

Here, we showed that 4-to-5 different classes of FDA-approved antibiotics can be used to selectively target CSCs, across multiple tumor types. Mechanistically, these antibiotics converge on three main mitochondrial targets, as summarized in Figure 15. Thus, molecular disruption of mitochondrial biogenesis or OXPHOS would be a novel therapeutic strategy for the eradication of CSCs. As a result, our findings have broad implications for the initiation of new clinical trials, for the re-purposing of antibiotics for the treatment of various cancer types, including "pre-malignant" and advanced metastatic disease.

This new therapeutic strategy takes advantage of the manageable side-effects of these antibiotics, which affect eukaryotic mitochondria, although these compounds are currently used for the broad-spectrum treatment of bacterial and parasitic infectious diseases.

In this regard, doxycycline is relatively attractive as a new anti-cancer agent, as it has a long half-life systemically and has been used successfully for the longterm treatment of patients with urinary tract infections (UTI), prostatitis or acne, for extended periods of time,

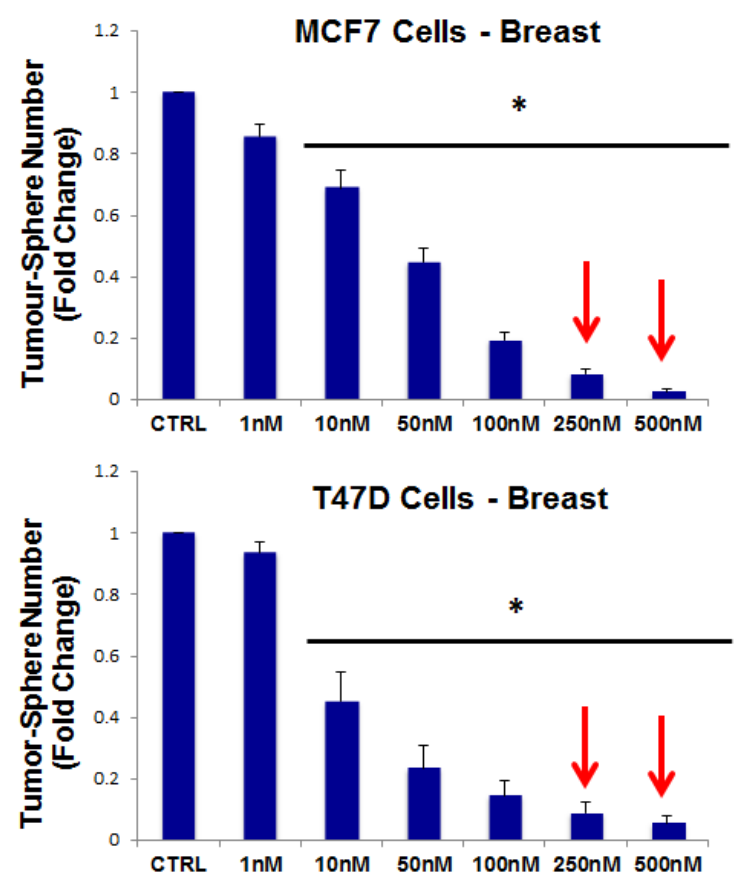

Figure 9: Pyrvinium pamoate dose-dependently inhibits tumor-sphere formation in MCF7 and T47D cells, two commonly used $\mathbf{E R}(+)$ breast cancer cell lines. Pyrvinium pamoate was initially tested over the range of $1 \mathrm{nM}$ to $500 \mathrm{nM}$. Note that $250 \mathrm{nM}$ and $500 \mathrm{nM}$ were the most effective. $(*) \mathrm{p}<0.001$. of up to 4-to-6 months or more (200 mg per day). Doxycycline also encourages the growth of normal stem cells, has anti-inflammatory properties, and even increases lifespan, in certain experimental contexts [12-14]. Thus, the toxic side effects of anti-cancer therapy would be minimized.

Doxycycline has also been used in human tumor xenografts and other animal models to significantly reduce tumor burden and even metastatic cancer cell growth [1520]. For example, in pancreatic tumor xenografts (with PANC-1 cells), doxycycline treatment reduced tumor growth by $\sim 80 \%$ [20]. In a xenograft model of breast cancer bone metastasis (with MDA-MB-231 cells), doxycycline treatment reduced bone and bone-associated soft-tissue tumor mass by $>60 \%$ and $\sim 80 \%$, respectively [19]. However, its anti-cancer activity was attributed to the inhibition of matrix-metalloproteinases (MMPs), rather than the targeting of mitochondrial biogenesis, and doxycycline has not been previously implicated in the selective eradication of cancer stem cells [15-20].

Our results are consistent with the previous finding that metformin, a widely used anti-diabetic drug, which functions as a mitochondrial inhibitor, can also be used to selectively target CSCs $[21,22]$. Metformin functionally inhibits OXPHOS by targeting complex I of the electron transport chain and can even induce lactic acidosis, as a lethal side effect $[21,22]$. As a result, the use of antibiotics, such as doxycycline, may provide a safer and far more effective alternative to anti-cancer therapy with metformin.

Our global phenotypic approach to target cancer as a single disease of stemness, may also help to avoid drug resistance. We speculate that genetic changes (oncogenic mutations, amplifications/deletions, and tumor suppressor loss) all converge on "stemness" in tumor-initiating CSCs (Figure 16), driving tumor recurrence, metastasis and drug resistance. Thus, it would be advantageous to phenotypically target "stemness" directly, instead of targeting individual genetic changes, in different cancer types. This would allow the treatment of cancer in a mutation-independent fashion.

Finally, recent clinical trials with doxycycline and azithromycin (intended to treat cancer-associated infections, but not cancer cells) both show positive therapeutic effects in cancer patients, although their selective effects on eradicating cancer stem cells were not yet known or appreciated [23-26]. These trials were performed on advanced or treatment-resistant patients with B-cell lymphoma (doxycycline) or lung cancer (azithromycin), respectively [23-26]. For example, in lung cancers, azithromycin significantly increased 1-year patient survival from $45 \%$ to $75 \%$, an $\sim 1.7$-fold increase [26]. Interestingly, it was noted that even lymphoma patients that were "bacteria-free" benefited from only a 3-week course of doxycycline therapy, and showed complete remission of the disease [27]. These results 


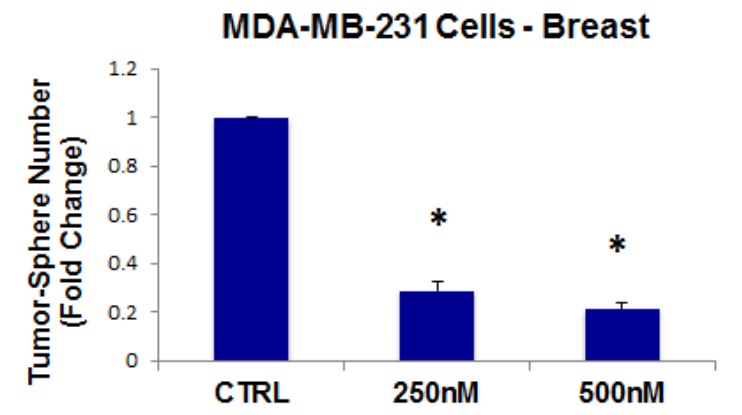

ES2 Cells - Ovarian

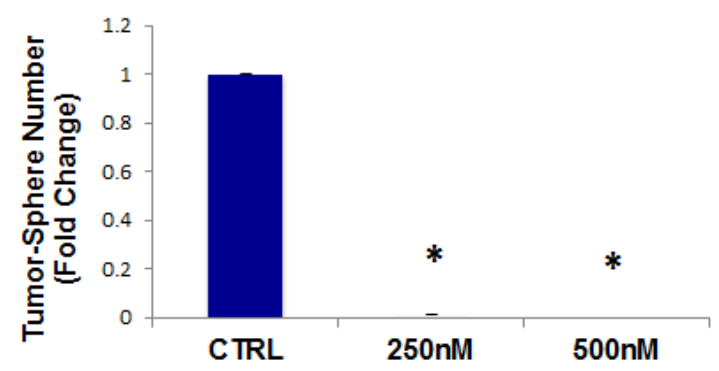

B

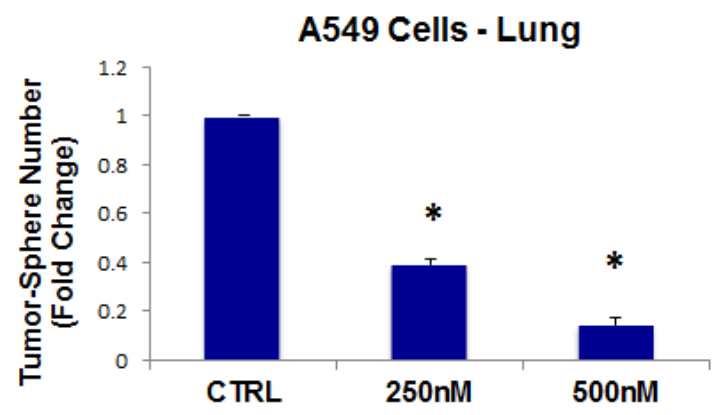

PC3 Cells - Prostate

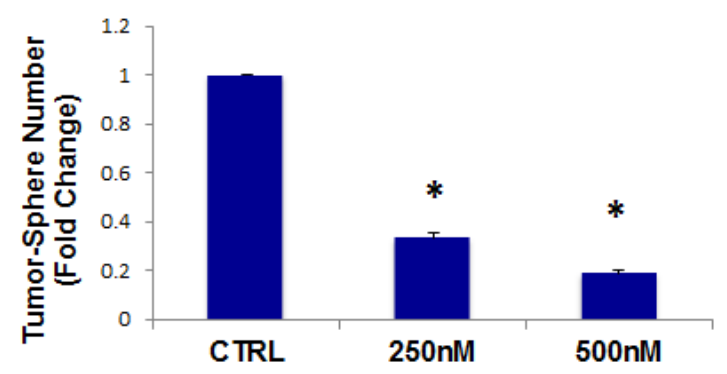

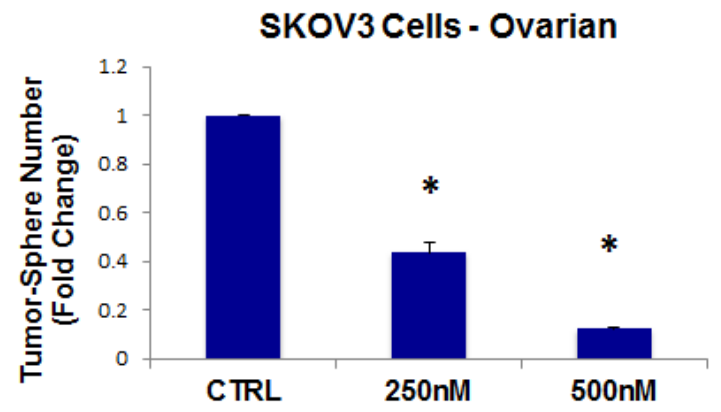

Tov21G Cells - Ovarian

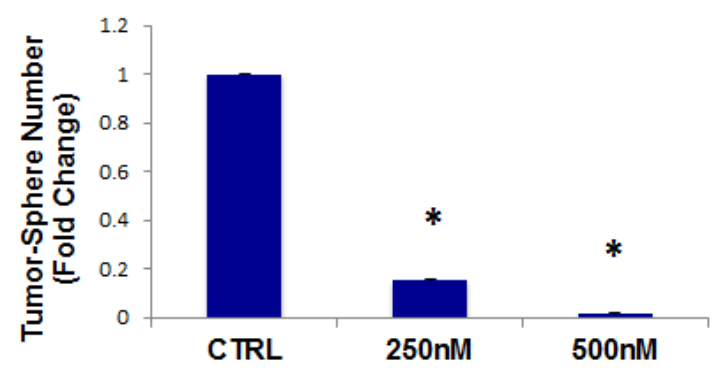

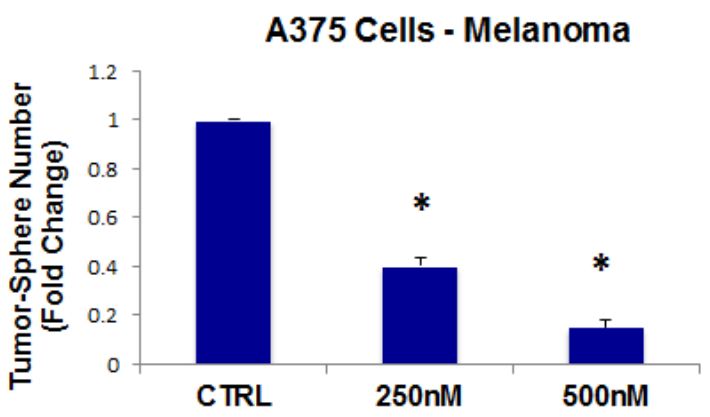

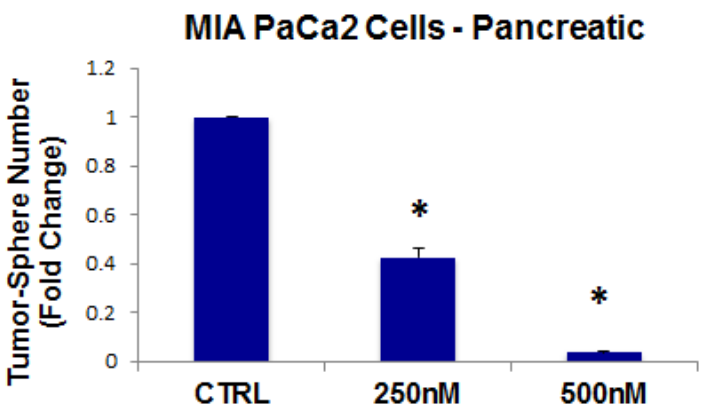

Figure 10: Pyrvinium pamoate inhibits tumor-sphere formation in eight other cell lines, derived from diverse cancer types. For simplicity, the efficacy of pyrvinium pamoate was tested at a concentration of $250 \mathrm{nM}$ and $500 \mathrm{nM}$. $(*) \mathrm{p}<0.001$. (A) ER(-) breast [MDA-MB-231] and ovarian cancer cell lines [SKOV3, ES2, Tov21G] (B) Lung [A549], prostate [PC3], melanoma [A375], and pancreatic [MIA PaCa2]. 
A

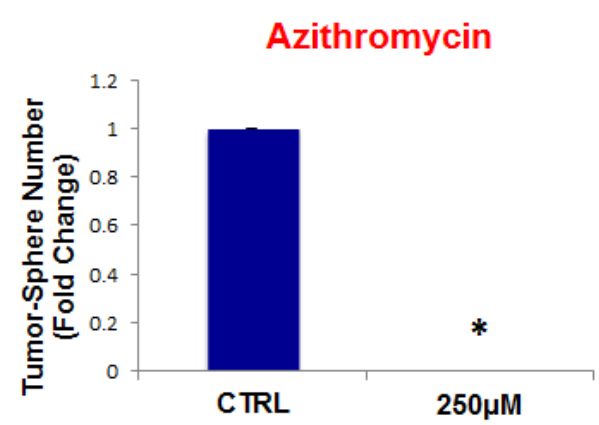

Tigecycline

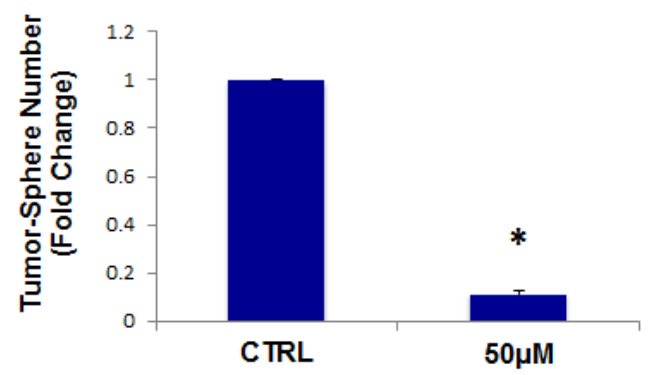

B

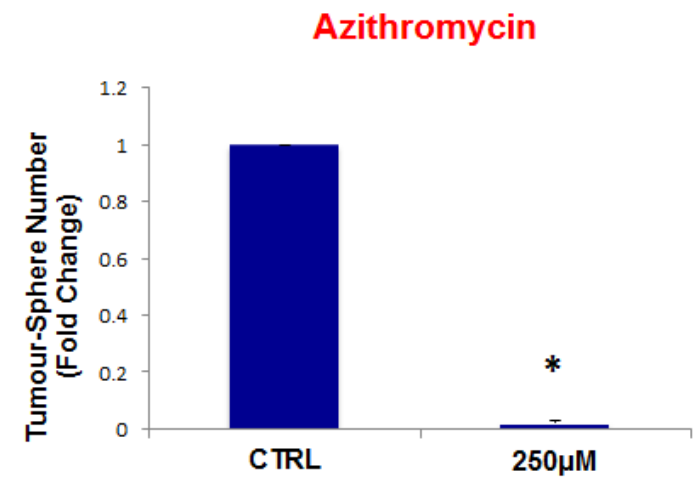

Tigecycline

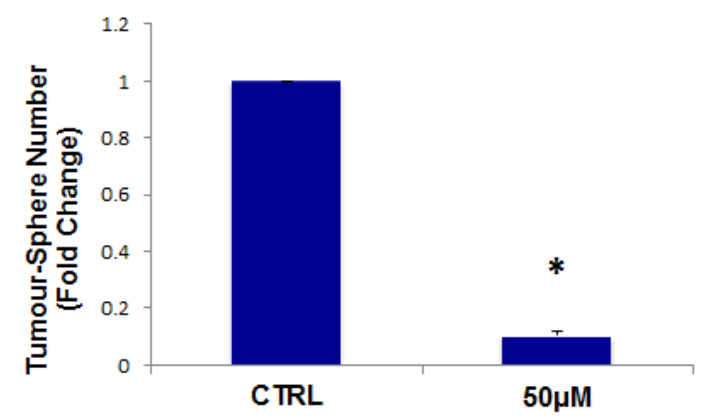

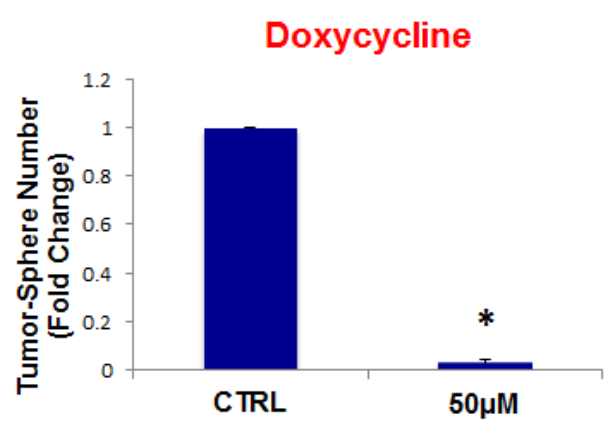

PP

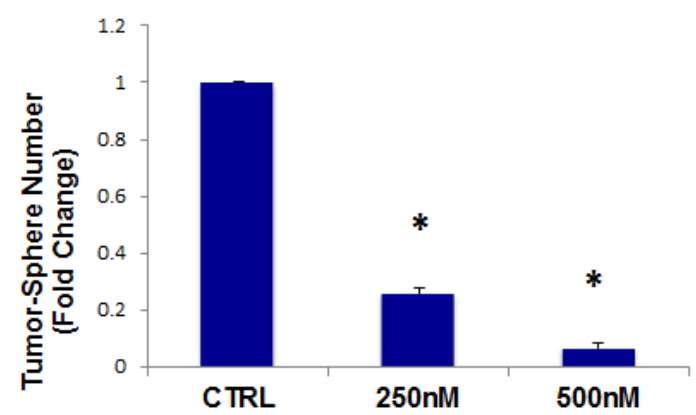

Doxycycline

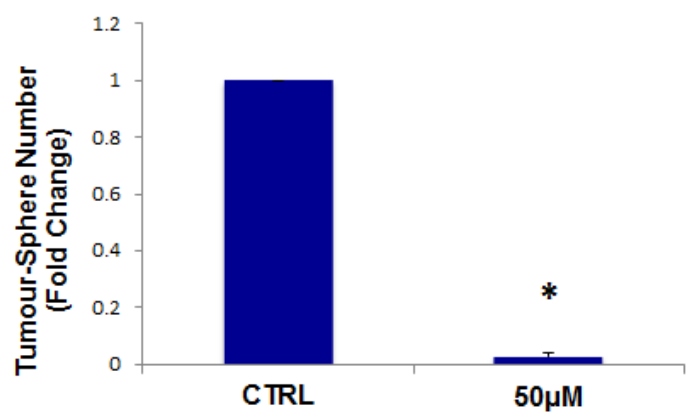

PP

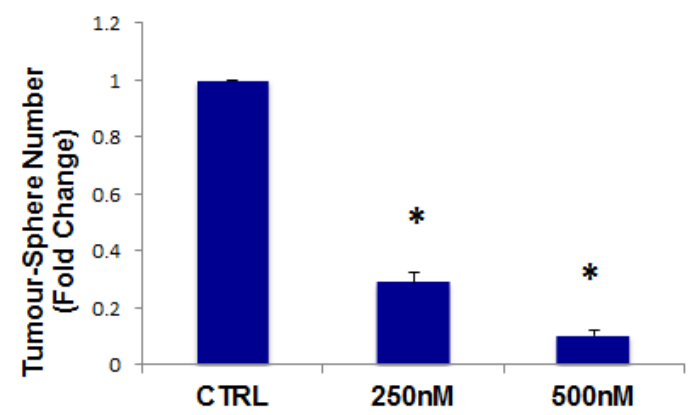

Figure 11: Four FDA-approved antibiotic classes also inhibit tumor-sphere formation in DCIS and glioblastoma cell lines. Antibiotics were tested at the following concentrations: azithromycin $(250 \mu \mathrm{M})$, doxycycline $(50 \mu \mathrm{M})$, tigecycline $(50 \mu \mathrm{M})$, and pyrvinium pamoate $(250$ and $500 \mathrm{nM})$. Note that all four antibiotics classes inhibit tumor-sphere formation, in both DCIS and glioblastoma cell lines. (A) DCIS.com; (B) U-87 MG. $\left(^{*}\right) \mathrm{p}<0.001$. 
A

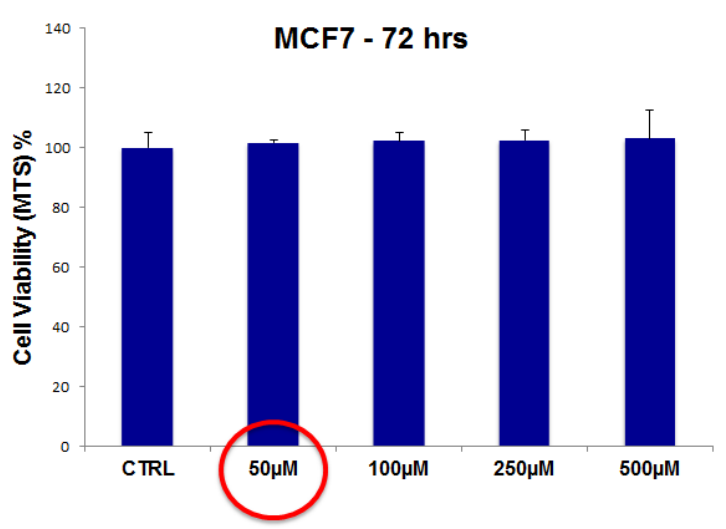

B

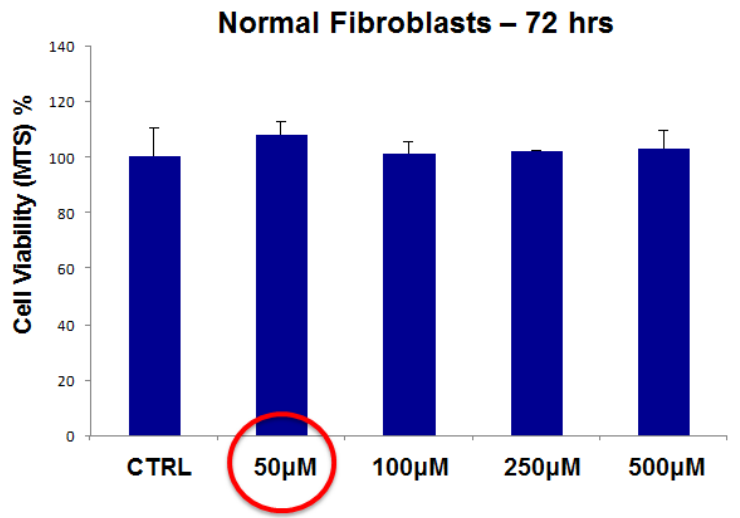

Figure 12: Doxycycline does not affect the viability of "bulk" cancer cells or normal fibroblasts. (A) MCF7 cell monolayers; (B) hTERT-BJ1 skin fibroblasts. Viability was quantitatively measured using the MTS assay. Doxcycline was tested over the range of $50 \mu \mathrm{M}$ to $500 \mu \mathrm{M}$, with little or no effects on cell viability.
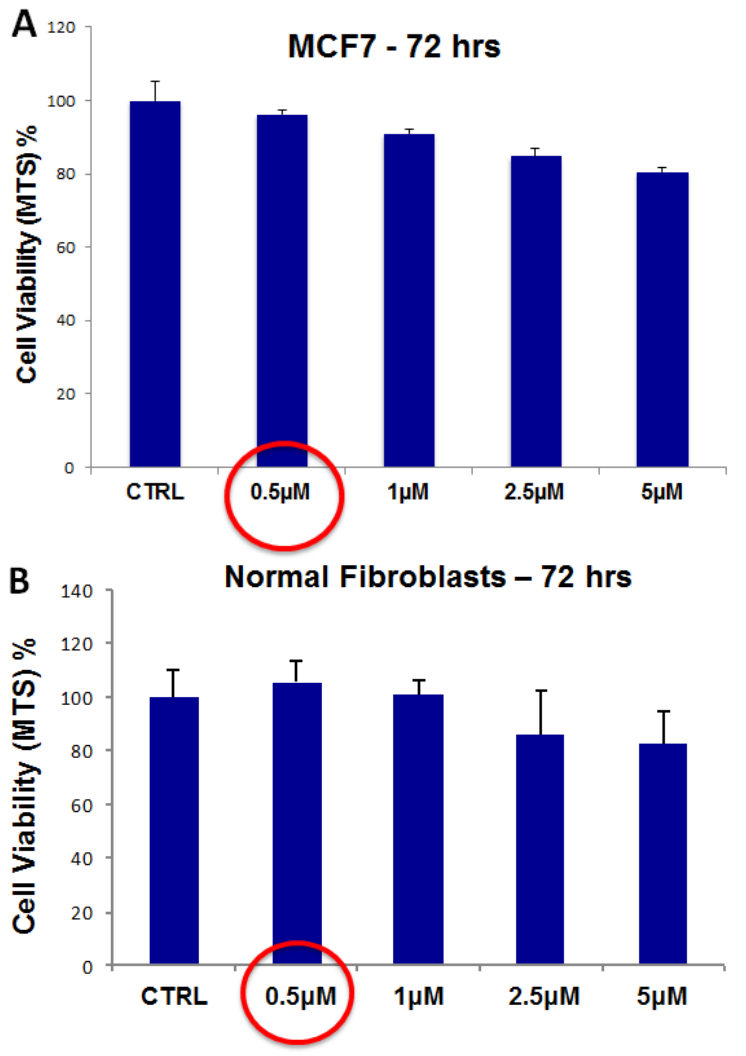

Figure 13: Pyrvinium pamoate does not affect the viability of "bulk" cancer cells or normal fibroblasts. (A) MCF7 cell monolayers; (B) hTERT-BJ1 skin fibroblasts. Viability was quantitatively measured using the MTS assay. Pyrvinium pamoate was tested over the range of $500 \mathrm{nM}$ to 5 $\mu \mathrm{M}$, with little or no effects on cell viability.

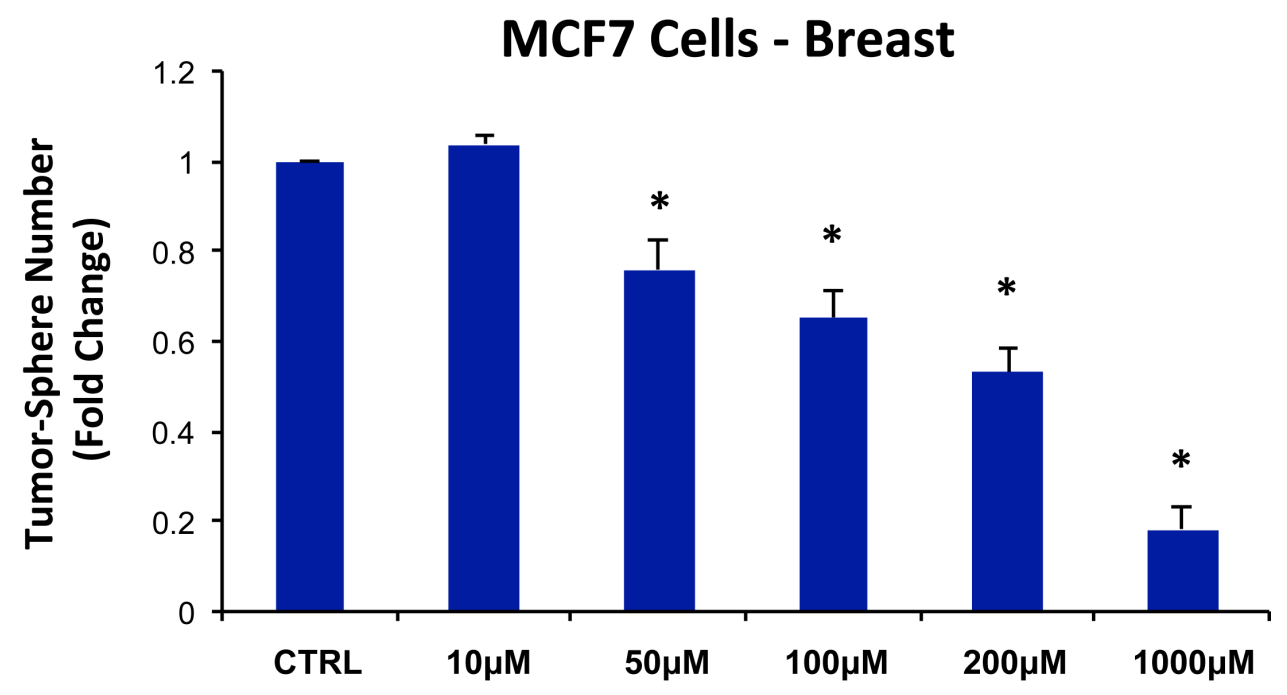

Figure 14: Chloramphenicol dose-dependently inhibits tumor-sphere formation in MCF7 cells. We determined the ability of chloramphenicol to inhibit mammo-sphere formation using MCF7 cells, over a range of concentrations from $10 \mu \mathrm{M}$ to $1 \mathrm{mM}$. Interestingly, in MCF7 cells, chloramphenicol inhibited mammo-sphere formation with an IC-50 of $\left.\sim 200 \mu \mathrm{M} .{ }^{*}\right) \mathrm{p}<0.002$. 
suggest that the antibiotic's therapeutic effects were actually infection-independent.

Thus, future clinical trials for testing the efficacy of mitochondrially-targetd antibiotics in multiple cancer types are now clearly clinically warranted. In this regard, a clinical trial with doxycycline in patients with advanced breast cancer and bone metastasis is ongoing: https:// clinicaltrials.gov/ct2/show/NCT01847976. Secondly, a trial of doxycycline in relapsed patients with nonhodgkin's lymphoma has also been initiated: https:// clinicaltrials.gov/ct2/show/NCT02086591. Interestingly, the A375 human melanoma cell line harbors the B-RAF(V600E) mutation and we showed that CSCs derived from this cell line are highly-sensitive to all four of the antibiotics that we tested (azithromcyin, doxycycline, tigecycline, and pyrvinium pamoate). As such, additional cellular studies, and new clinical trails in melanoma patients with B-RAF mutations, may be indicated, to explore the use of antibiotics.

SUMMARY

5 Drug Classes

3 Molecular Targets

1. Erythromycins

2. Chloramphenicol

3. Tetracyclines

4. Glycylcyclines

5. Pyrvinium pamoate
39S Large Mito Ribosome

39S Large Mito Ribosome

28S Small Mito Ribosome

28S Small Mito Ribosome

Mito OXPHOS

Figure 15: Summary of drug discovery. The five-classes of antibiotics that we tested are summarized here, along with their three corresponding molecular targets, which all converge on mitochondrial biogenesis or OXPHOS.

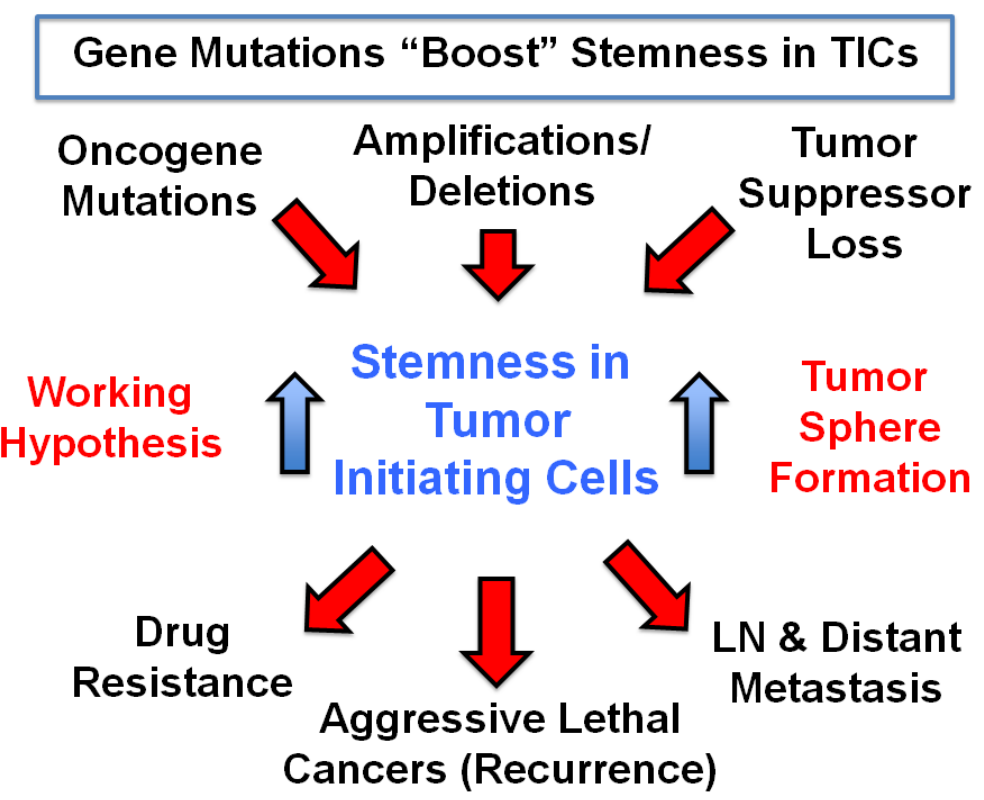

Figure 16: A mutation-independent approach to cancer therapy. We speculate that genetic changes (oncogenic mutations, amplifications/deletions, and tumor suppressor loss) all converge on "stemness" in tumor-initiating cells, driving tumor recurrence, metastasis and drug resistance. Thus, it would be advantageous to phenotypically target "stemness" directly, instead of targeting individual genetic changes, in different cancer types. This would allow the treatment of cancer as a single disease of stemness, in a mutationindependent fashion. 


\section{MATERIALS AND METHODS}

\section{Materials}

Cancer cell lines were purchased from the ATCC or other commercially available sources. Antibiotics were all obtained commercially from Sigma-Aldrich. Gibco-brand cell culture media (DMEM/F12) was purchased from Life Technologies.

\section{Tumor-sphere Culture}

A single cell suspension was prepared using enzymatic (1x Trypsin-EDTA, Sigma Aldrich, \#T3924), and manual disaggregation (25 gauge needle) to create a single cell suspension. Cells were plated at a density of 500 cells $/ \mathrm{cm} 2$ in mammosphere medium (DMEM-F12/ B27/20ng/ml EGF/PenStrep) in non-adherent conditions, in culture dishes coated with (2-hydroxyethylmethacrylate) (poly-HEMA, Sigma, \#P3932) [28]. Cells were grown for 5 days and maintained in a humidified incubator at $37^{\circ} \mathrm{C}$ at an atmospheric pressure in $5 \%(\mathrm{v} / \mathrm{v})$ carbon dioxide/air. After 5 days for culture, spheres $>50 \mu \mathrm{m}$ were counted using an eye piece graticule, and the percentage of cells plated which formed spheres was calculated and is referred to as percentage tumor-sphere formation (TSF), and was normalized to one $(1=100 \% \mathrm{TSF})$ [28]. All experiments were performed in triplicate, three times independently, such that each data point represents the average of 9 replicates.

For tumor-sphere assays, all cell line derived spheroids (MCF7, T47D, MDA-MB-231, DCIS.com, SKOV3, Tov21G, ES2, and A549) were counted at 5 days after plating, with the exception of PC3, A375, and MIA $\mathrm{PaCa} 2$ cells, which were counted at 3 days post-seeding. Finally, U-87 MG spheroids were counted at 7 days. All data shown are the mean $+/$ - the standard error of the mean (SEM).

\section{ACKNOWLEDGEMENTS}

We thank the University of Manchester for providing start-up funds that contributed to the success of this study. In addition, the research laboratories of Professor Lisanti and Dr. Sotgia were supported by funding schemes from the European Union (ERC Advanced Grant), Breakthrough Breast Cancer (BBC), and the Manchester Cancer Research Centre (MCRC). Ubaldo MartinezOutschoorn was supported by the National Cancer Institute (NCI) of the National Institutes of Health (NIH), under Award Number K08 CA -175193-01A1.

\section{REFERENCES}

1. Cancer Genome Atlas Network. Comprehensive molecular portraits of human breast tumours. Nature. 2012; 490(7418): 61-70.

2. Cancer Genome Atlas Research Network. Comprehensive molecular profiling of lung adenocarcinoma. Nature. 2014; 511(7511): 543-50.

3. Cancer Genome Atlas Research Network. Integrated genomic analyses of ovarian carcinoma. Nature. 2011; 474(7353): 609-15.

4. Desmedt C1, Voet T, Sotiriou C, Campbell PJ. Nextgeneration sequencing in breast cancer: first take home messages. Curr Opin Oncol. 2012; 24(6): 597-604.

5. Stingl J, Caldas C. Molecular heterogeneity of breast carcinomas and the cancer stem cell hypothesis. Nat Rev Cancer. 2007; 7(10): 791-9.

6. Flaherty KT. BRAF inhibitors and melanoma. Cancer J. 2011; 17(6): 505-11.

7. Girotti MR, Lopes F, Preece N, Niculescu-Duvaz D, Zambon A, Davies L, Whittaker S, Saturno G, Viros A, Pedersen M, Suijkerbuijk BM, Menard D, McLeary R, Johnson L, Fish L, Ejiama S, Sanchez-Laorden B, Hohloch J, Carragher N, Macleod K, Ashton G, Marusiak AA, Fusi A, Brognard J, Frame M, Lorigan P, Marais R, Springer C. Paradox-Breaking RAF Inhibitors that Also Target SRC Are Effective in Drug-Resistant BRAF Mutant Melanoma. Cancer Cell. 2015 ;27(1): 85-96.

8. Tomasetti C, Vogelstein B. Variation in cancer risk among tissues can be explained by the number of stem cell divisions. Science. 2015; 347(6217): 78-81.

9. Lamb R, Harrison H, Hulit J, Smith DL, Lisanti MP, Sotgia F. Mitochondria as new therapeutic targets for eradicating cancer stem cells: Quantitative proteomics and functional validation via MCT1/2 inhibition. Oncotarget. 2014; 5(22): 11029-37.

10. Zimorski V, Ku C, Martin WF, Gould SB. Endosymbiotic theory for organelle origins. Curr Opin Microbiol. 2014; $22 \mathrm{C}: 38-48$.

11. Degli Esposti M, Chouaia B, Comandatore F, Crotti E, Sassera D, Lievens PM, Daffonchio D, Bandi C. Evolution of mitochondria reconstructed from the energy metabolism of living bacteria. PLoS One. 2014; 9(5): e96566.

12. Chang MY, Rhee YH, Yi SH, Lee SJ, Kim RK, Kim H, Park CH, Lee SH. Doxycycline enhances survival and selfrenewal of human pluripotent stem cells. Stem Cell Reports. 2014; 3(2): 353-64.

13. Houtkooper RH, Mouchiroud L, Ryu D, Moullan N, Katsyuba E, Knott G, Williams RW, Auwerx J. Mitonuclear protein imbalance as a conserved longevity mechanism. Nature. 2013; 497(7450): 451-7.

14. Krakauer T1, Buckley M. Doxycycline is anti-inflammatory and inhibits staphylococcal exotoxin-induced cytokines and chemokines. Antimicrob Agents Chemother. 2003; 47(11): 
3630-3.

15. Shen LC, Chen YK, Lin LM, Shaw SY. Anti-invasion and anti-tumor growth effect of doxycycline treatment for human oral squamous-cell carcinoma--in vitro and in vivo studies. Oral Oncol. 2010; 46(3): 178-84.

16. Foroodi F, Duivenvoorden WC, Singh G. Interactions of doxycycline with chemotherapeutic agents in human breast adenocarcinoma MDA-MB-231 cells.Anticancer Drugs. 2009; 20(2): 115-22.

17. Sapadin AN, Fleischmajer R. Tetracyclines: nonantibiotic properties and their clinical implications. J Am Acad Dermatol. 2006; 54(2): 258-65.

18. Saikali Z1, Singh G. Doxycycline and other tetracyclines in the treatment of bone metastasis. Anticancer Drugs. 2003; 14(10): 773-8.

19. Duivenvoorden WC, Popović SV, Lhoták S, Seidlitz E, Hirte HW, Tozer RG, Singh G. Doxycycline decreases tumor burden in a bone metastasis model of human breast cancer. Cancer Res. 2002; 62(6): 1588-91.

20. Son K, Fujioka S, Iida T, Furukawa K, Fujita T, Yamada $\mathrm{H}$, Chiao PJ, Yanaga K. Doxycycline induces apoptosis in PANC-1 pancreatic cancer cells. Anticancer Res. 2009; 29(10): 3995-4003.

21. Rattan R, Ali Fehmi R, Munkarah A. Metformin: an emerging new therapeutic option for targeting cancer stem cells and metastasis. J Oncol. 2012; 2012: 928127.

22. Hirsch HA, Iliopoulos D, Tsichlis PN, Struhl K. Metformin selectively targets cancer stem cells, and acts together with chemotherapy to block tumor growth and prolong remission. Cancer Res. 2009; 69(19): 7507-11.

23. Han JJ, Kim TM, Jeon YK, Kim MK, Khwarg SI, Kim CW, Kim IH, Heo DS. Long-term outcomes of first-line treatment with doxycycline in patients with previously untreated ocular adnexal marginal zone B cell lymphoma. Ann Hematol. 2014 Oct 24. [Epub ahead of print].

24. Ferreri AJ, Dolcetti R, Magnino S, Doglioni C, Cangi MG, Pecciarini L, Ghia P, Dagklis A, Pasini E, Vicari N, Dognini GP, Resti AG, Ponzoni M. A woman and her canary: a tale of chlamydiae and lymphomas. J Natl Cancer Inst. 2007; 99(18): 1418-9.

25. Ferreri AJ, Ponzoni M, Guidoboni M, De Conciliis C, Resti AG, Mazzi B, Lettini AA, Demeter J, Dell’Oro S, Doglioni C, Villa E, Boiocchi M, Dolcetti R. Regression of ocular adnexal lymphoma after Chlamydia psittaci-eradicating antibiotic therapy. J Clin Oncol. 2005; 23(22): 5067-73.

26. Chu DJ, Yao DE, Zhuang YF, Hong Y, Zhu XC, Fang ZR, $\mathrm{Yu}$ J, Yu ZY. Azithromycin enhances the favorable results of paclitaxel and cisplatin in patients with advanced nonsmall cell lung cancer. Genet Mol Res. 2014; 13(2): 2796805.

27. Ferreri AJ1, Ponzoni M, Guidoboni M, Resti AG, Politi LS, Cortelazzo S, Demeter J, Zallio F, Palmas A, Muti G, Dognini GP, Pasini E, Lettini AA, Sacchetti F, De Conciliis C, Doglioni C, Dolcetti R. Bacteria-eradicating therapy with doxycycline in ocular adnexal MALT lymphoma: a multicenter prospective trial. J Natl Cancer Inst. 2006; 98(19): 1375-82.

28. Shaw FL, Harrison H, Spence K, Ablett MP, Simoes BM, Farnie G, Clarke RB. A detailed mammosphere assay protocol for the quantification of breast stem cell activity. $\mathrm{J}$ Mammary Gland Biol Neoplasia. 2012; 17(2): 111-117. 